\title{
Caractérisation et estimation du gonflement des argiles algériennes, cas des argiles de Médéa
}

\author{
MEDJNOUN Amal ${ }^{1}$, BAHAR Ramdane ${ }^{2} \&$ KHIATINE Mohamed ${ }^{3}$ \\ ${ }^{1}$ Université Mouloud Mammeri de Tizi-Ouzou, Laboratoire de Géomatériaux, Environnement et Aménagement, Tizi-Ouzou, \\ Algérie \\ ${ }^{2}$ Université des Sciences et de la Technologie Houari Boumediene, Faculté de Génie Civil, LEEGO, Bab Ezzouar, Alger, \\ Algérie \\ ${ }^{3}$ Construction and Testing Engineering Laboratory, Alger, Algérie
}

\begin{abstract}
Résumé. Le matériau argileux, dur et cassant lorsqu'il est asséché, présente la particularité de voir sa consistance se modifier en fonction de sa teneur en eau. Un certain degré d'humidité peut le transformer en un matériau plastique et malléable. Ces modifications de consistance peuvent s'accompagner, en fonction de la structure particulière de certains minéraux argileux, de variations de volume plus ou moins conséquentes qui se manifestent soit sous forme d'une forte augmentation de volume (phénomène de gonflement) lorsque la teneur en eau augmente ou de rétrécissement (phénomène de retrait) en période de déficit pluviométrique marqué. Ce comportement est lié essentiellement à certains paramètres environnementaux de ces sols à l'exemple de la géologie et de la lithologie du site, du climat, etc. La prévision du potentiel de gonflement des sols est possible moyennant certaines méthodes empiriques basées sur des paramètres d'identification tels que la plasticité et la granulométrie déterminées lors des campagnes de reconnaissance. Une fois le caractère de gonflement pressenti dans la phase de reconnaissance préliminaire, une estimation de l'amplitude et de la pression de gonflement peut être établie à partir des corrélations, qui tendent à mettre en relation les paramètres de gonflement avec les paramètres géotechniques issus des essais d'identification. C'est dans cet aspect que s'inscrit cet article en traitant le cas d'un site algérien. Les résultats obtenus montrent une concordance entre les méthodes empiriques appliquées. Ces résultats sont aussi comparés avec ceux obtenus des essais de gonflement à l'oedométrique. L'évolution de la structure argileuse au cours de retraitgonflement est suivie dans l'objectif de comprendre le mécanisme de gonflement.
\end{abstract}

Mots clés : Argiles gonflantes, identification, prévision, estimation, évolution de gonflement.

\begin{abstract}
The clayey material hard and brittle when dry, presents the particularity of changing its consistence as a function of its water content. A certain percentage of moisture may transformed it into a plastic and manageable material. Depending on the particular structure of clay minerals, these modifications of consistence may be accompanied by more or less substantial volume variations, manifested, either, as a sharp increase of volume (swelling phenomenon) when the water content increases or shrinkage (shrinkage phenomenon) in period of marked rainfall. This behavior is essentially linked to some of environmental parameters of these soils, for example: the geology and lithology of the site, the climate, etc. The prediction of swelling potential of soil is possible with empirical methods that based on identification parameters, such as plasticity and particle size, determined during the reconnaissance campaigns. Once the character of the swelling sensed in preliminary recognition phase, an estimate of the amplitude and the pressure of swelling can be established by correlations that tend to link the swelling parameters with geotechnical parameters obtained from identification tests. It is in this aspect that is inscribed this article which treating the case of an Algerian Site. The results show a concordance between the deferent empirical methods applied. These results are compared with those obtained for the oedometric swelling tests. The evolution of the clay structure during shrinkage and swelling is followed with the aim of understanding the mechanism of swelling.
\end{abstract}

Keywords: Clay swelling; Identification; Prediction; Estimation; Swelling evolution. 


\section{Introduction}

La reconnaissance du sol gonflant se fait progressivement tout en se basant sur des données existantes et d'autres recherchées. $\mathrm{La}$ formation argileuse est connue pour sa sensibilité aux variations de la teneur en l'eau, c'est la raison pour la quelle le phénomène de gonflement se manifeste dans les régions à climat aride et semi aride. L'importance de ce phénomène dépend essentiellement de la quantité des minéraux gonflants. Son étude se base sur plusieurs aspects à savoir: géologique, climatologique et géotechnique. Cet article présente une étude estimative des argiles d'un site soupçonné gonflant dans la région de Médéa, cet étude se base essentiellement sur les essais physiques, chimiques et mécaniques réalisés sur des échantillons intacts et remaniés. Une classification de potentiel de gonflement à été effectuée en utilisant des méthodes indirectes [2, 3, 4, 6, 7, 9] et directes existantes dans la littérature. L'étude du mécanisme de gonflement à l'échelle microscopique et macroscopique est également abordée.

\section{Description du site}

La Wilaya de Médéa se situe à environ 80 kilomètres au Sud d'Alger et au cœur de l'Atlas Tellien. Elle est caractérisée par une altitude élevée et un relief accidenté. La zone étudiée est appelée : le site des 8000 places pédagogiques (Fig.1), elle est située au Nord-Est du chef-lieu de la Wilaya de Médéa, terrain agricole (vignoble) et s'étend sur une superficie de $11904 \mathrm{~m}^{2}$. L'assiette se trouve au lieu-dit Haouch Beyzid, commune de Médéa (Axe Médéa-Ouzera). La position géographiques du centre du site (lot $\left.n^{\circ} 01\right)$ selon les coordonnées UTM est : $\mathrm{X}=483316.98$ et $\mathrm{Y}=$ 4014 434.14. L'altitude moyenne du site est $Z=847$ mètres.

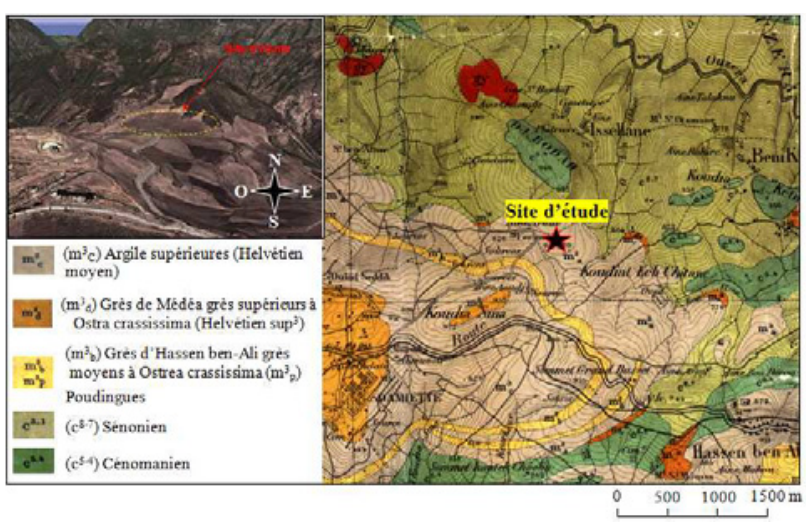

Fig. 1. Extrait de la carte géologique et topographique de la région de Médéa, feuille ${ }^{\circ} 86$, échelle 1/50000.

\subsection{Aspect géologique et minéralogique}

La zone étudiée fait partie du bassin de Médéa dont le plateau gréseux surplomb. L'examen des affleurements de surface et de la carte géologique à l'échelle régionale, montre l'existence d'un faciès assez homogène sur l'ensemble du site: Il s'agit de la formation marneuse argileuse de l'Helvétien (miocène moyen), série grisâtre et très épaisse (plus de $150 \mathrm{~m}$ ). Cet épaisse série constitue le substratum des formations gréseuses sableuses du plateau de Médéa.

L'aspect microscopique de la formation argileuse marneuse est présenté dans la figure 2. L'observation au MEB montre que la microstructure est formée par une matrice argileuse relativement homogène et compacte, elle montre aussi la disposition parallèle des feuillets argileux.

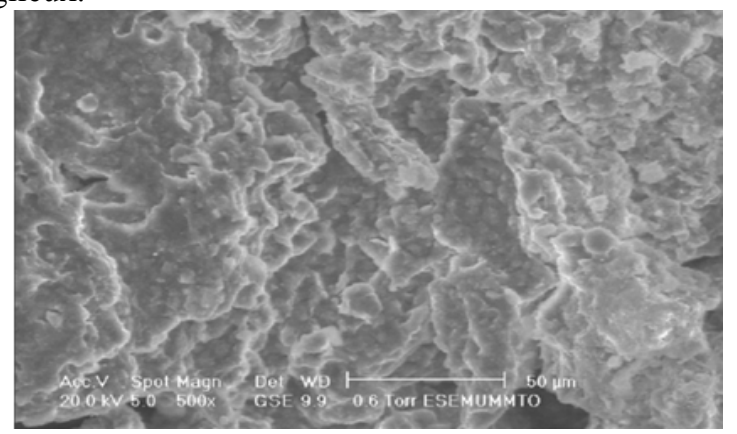

Fig. 2. Image au MEB d'un échantillon intact d'argile de Médéa.

\subsection{Aspect climatologique du site}

Sur le plan climatique, la région de Médéa qui est située à $80 \mathrm{~km}$ de la côte ne bénéficie pas énormément de l'influence maritime, toutefois son régime climatique reste régional subhumide littoral avec une tendance vers un climat continental sur la partie sud Atlassique.

La courbe mensuelle des précipitations, montre un maximum, très accusé entre les mois de décembre et janvier avec des valeurs supérieures à $120 \mathrm{~mm} /$ mois. Par contre, on a un minimum en juillet, période sèche, avec des valeurs inférieures à $10 \mathrm{~mm}$. La précipitation moyenne annuelle est de $700 \mathrm{~mm}$.

Le climat est chaud et sec en été ; La température atteint son maximum pendant les trois mois de cette saison avec un faible taux d'humidité variant entre 4 et $8 \%$. La Figure 3 montre l'existence d'un cycle d'humidificationséchage, se produisant chaque année depuis 1995 jusqu'à 2009 avec le déroulement des saisons, une saison pluvieuse et humide et une autre sèche et chaude

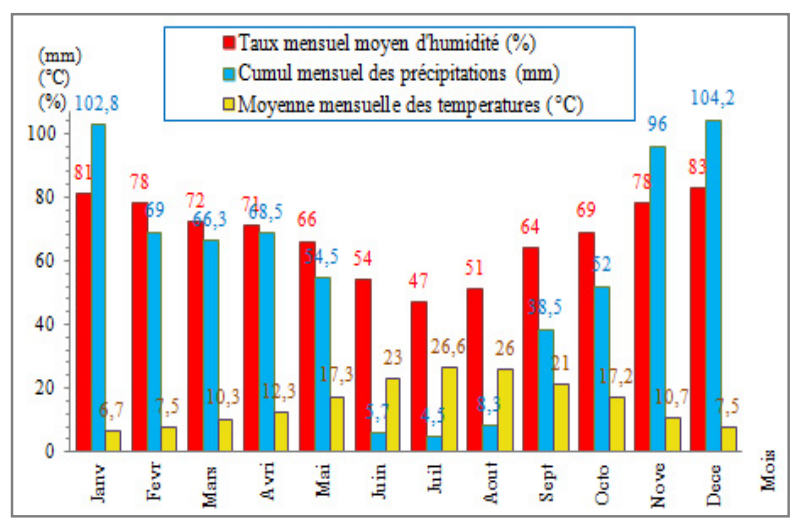

Fig. 3. Variation mensuelle moyenne des précipitations, humidités et températures dans la région de Médéa durant la période 1995 - 2009 (données de l'Office National Météorologique d'Alger, Algérie). 


\subsection{Aspect géotechnique}

Les échantillons intacts prélevés dans la couche d'argile marneuse au niveau du site ont été soumis à des essais d'identification physiques et chimiques. L'ensemble des résultats obtenus sont récapitulés dans le tableau 1. Les propriétés mesurées sont :

- Les limites d'Atterberg (limite de liquidité $\mathrm{w}_{\mathrm{L}}$, indice de plasticité $I_{p}$ )

- La granulométrie, caractérisée par le pourcentage des grains dont la dimension est inferieure à $2 \mu \mathrm{m}$ (proportion d'argile F2: $\%<2 \mu \mathrm{m}$ ).

- L'activité $\mathrm{A}_{\mathrm{c}}=\mathrm{I}_{\mathrm{p}} / \mathrm{F} 2$.

- La teneur en eau naturelle (w)

- Le poids volumique $\sec \left(\gamma_{\mathrm{d}}\right)$

- La valeur de bleu VB. Les valeurs de surface spécifique totale $\left(\mathrm{S}_{\mathrm{st}}\right)$ sont déduites de l'essai de bleu de méthylène par la formule suivante [8]:

$$
\mathrm{S}_{\mathrm{st}}\left[\mathrm{m}^{2} / \mathrm{g}\right]=21 \mathrm{VB}^{*}(\%<2 \mu \mathrm{m})
$$

La masse moléculaire étant égale à $373 \mathrm{~g}$ pour notre cas.

Les résultats obtenues à partir des essais physiques permettent de mettre en évidence un sol argileux (la fraction argileuse $>50 \%$ ) très plastique (fig. 4) d'une activité élevé (Ac >1,25). Le sol est dense et moyennement humide (tableau 1). Les valeurs de la surface spécifique varient entre 99 et $160 \mathrm{~m}^{2} / \mathrm{g}$, ces résultats indiquent que le site étudié est composé d'un mélange de minéraux argileux gonflant et non gonflant.

L'ensemble de ces résultats nous mènent à penser à une argile gonflante. En effet, le fait de soupçonner qu'un sol puisse être gonflant dès la campagne de reconnaissance préliminaire permet d'anticiper sur les campagnes de reconnaissance de confirmation en les adaptant à la nature du site [5].

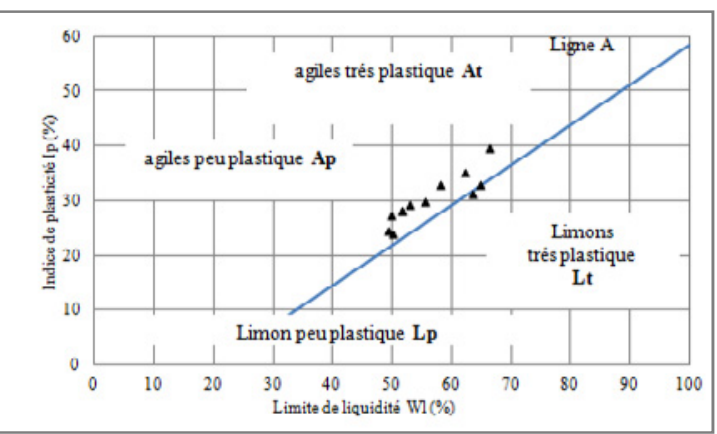

Fig. 4. Présentation des limites de consistances dans l'abaque de Casagrand.

Tableau 1. Résultats d'essais d'identification de l'argile de Médéa

\begin{tabular}{|c|c|c|c|c|c|c|c|}
\hline $\begin{array}{c}\text { Sondage } \\
\text { et } \\
\text { Prof }(\mathrm{m})\end{array}$ & $\begin{array}{c}\mathrm{W} \\
(\%)\end{array}$ & $\begin{array}{c}\gamma_{\mathrm{d}} \\
(\mathrm{kN} / \\
\left.\mathrm{m}^{3}\right)\end{array}$ & $\begin{array}{c}\mathrm{S}_{\mathrm{r}} \\
(\%)\end{array}$ & $A c$ & $\begin{array}{c}\%< \\
2 \\
\mu m\end{array}$ & $V B$ & $\begin{array}{c}S_{s t} \\
\left(\mathrm{~m}^{2} /\right. \\
g\end{array}$ \\
\hline $\begin{array}{c}\mathrm{E} 3 \\
2,4 / 2,6\end{array}$ & 27,3 & 15,2 & 98 & 2,0 & 62 & - & - \\
\hline $\begin{array}{c}\mathrm{E} 3 \\
4,2 / 4,6\end{array}$ & 20,9 & 17,4 & 100 & 2,3 & 55 & 6 & 125 \\
\hline $\begin{array}{c}\mathrm{S} 3 \\
5,5 / 6,0\end{array}$ & 22,8 & 17,1 & 100 & 2,2 & 56 & 4,8 & 102 \\
\hline
\end{tabular}

\begin{tabular}{|c|c|c|c|c|c|c|c|}
\hline $\begin{array}{c}\mathrm{E} 4 \\
2,6 / 3,0\end{array}$ & 27,3 & 15,2 & 88 & 1,8 & 53 & 5,6 & 117 \\
\hline $\begin{array}{c}\mathrm{E} 4 \\
3,3 / 3,6\end{array}$ & 17,4 & 17,9 & 95 & 1,9 & 52 & 6 & 126 \\
\hline $\begin{array}{c}\mathrm{E} 5 \\
5,0 / 5,5\end{array}$ & 21,2 & 16,9 & 99 & 1,7 & 56 & 7,4 & 156 \\
\hline $\begin{array}{c}\mathrm{E} 5 \\
6,2 / 7,0\end{array}$ & 21,8 & 17,3 & 100 & 2,0 & 62 & 6,8 & 142 \\
\hline $\begin{array}{c}\mathrm{E} 6 \\
4,5 / 4,8\end{array}$ & 17,0 & 19,8 & 93 & 1,6 & 56 & 5,6 & 119 \\
\hline $\begin{array}{c}\mathrm{E} 7 \\
2,4 / 2,8\end{array}$ & 20,5 & 17,0 & 96 & 2,1 & 60 & 7,7 & 161 \\
\hline $\begin{array}{c}\mathrm{E} 7 \\
5,0 / 5,5\end{array}$ & 18,3 & 16,4 & 97 & 1,3 & 40 & 6,5 & 136 \\
\hline $\begin{array}{c}\mathrm{E} 10 \\
4,7 / 5,0\end{array}$ & 30,5 & 15,0 & 100 & 1,3 & 52 & 6,7 & 142 \\
\hline $\begin{array}{c}\mathrm{E} 10 \\
8,6 / 8,9\end{array}$ & 26,3 & 16,0 & 100 & 1,5 & 51 & 4,7 & 99 \\
\hline
\end{tabular}

\section{Estimation du potentiel de gonflement}

\subsection{Méthode indirecte}

De nombreuses méthodes ont été proposées dans la littérature pour évaluer le potentiel de gonflement d'un sol à partir de la mesure des paramètres de plasticité et de granulométrie.

Pour les argiles présentées dans cet étude, le potentiel de gonflement est analysé à l'aide des méthodes de BRE, Royaume uni (1980), Chen (1988), Komornik \& David (1969), Seed \& al. (1962), Snethen (1984), Vijayvergiya \& Ghazzaly (1973) et Williams \& Donaldson (1980). Les résultats de la classification sont cités dans le tableau 2.

De ces résultats, on peut conclure que le caractère gonflant des sols examinés apparaît clairement à travers l'ensemble des classifications citées, les classifications basées sur la fraction argileuse ainsi que les limites d'Atterberg estiment gonflant le sol étudié et la classification [9] basée sur la valeur de bleu de méthylène l'estime moyennement gonflant.

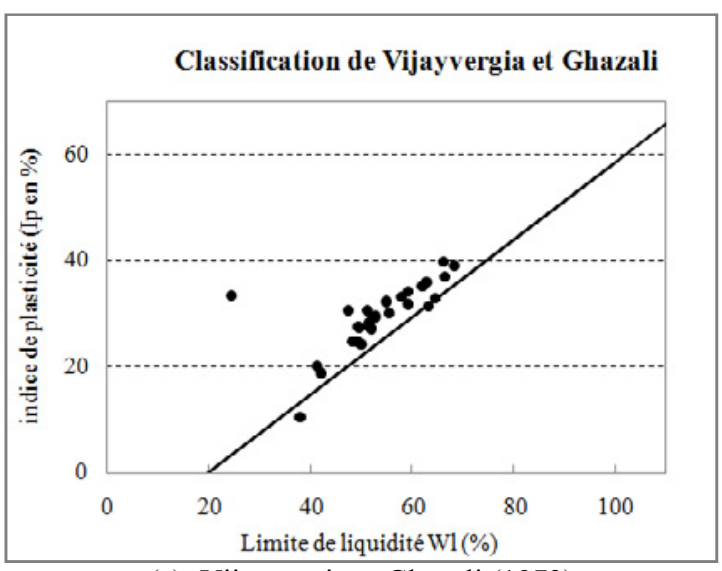

(a): Vijayvergia et Ghazali (1973) 


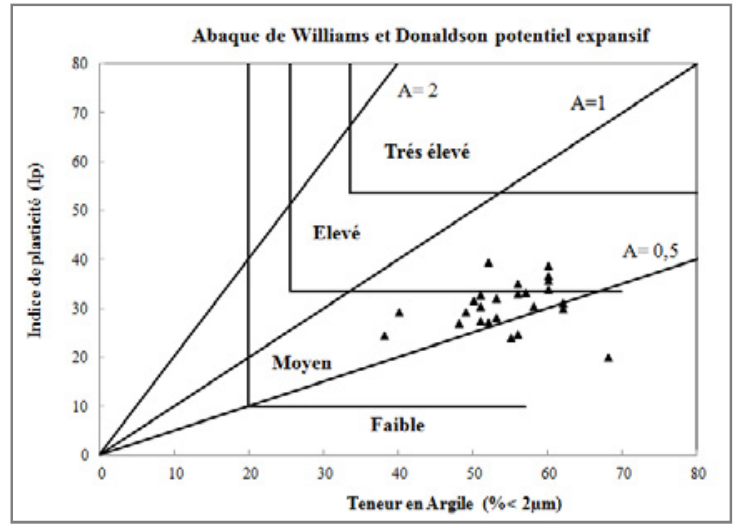

(b) : Williams et Donaldson (1980)

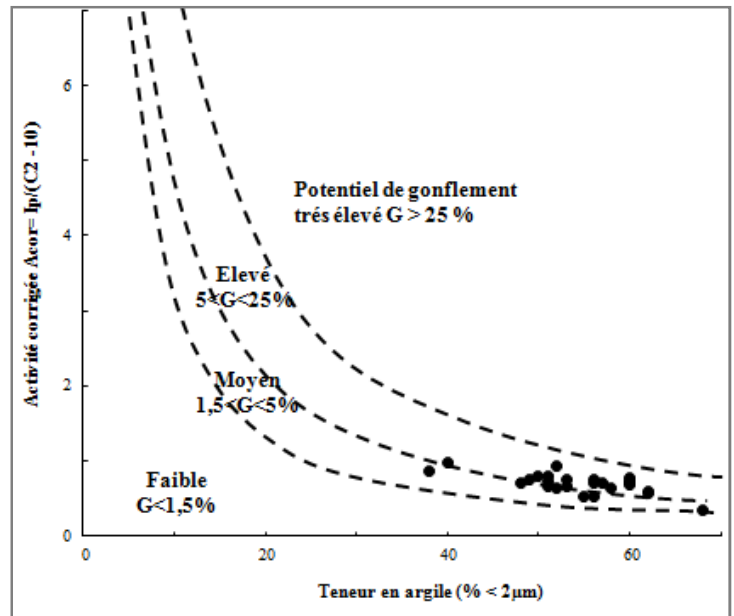

(c) : Seed et al. 1962

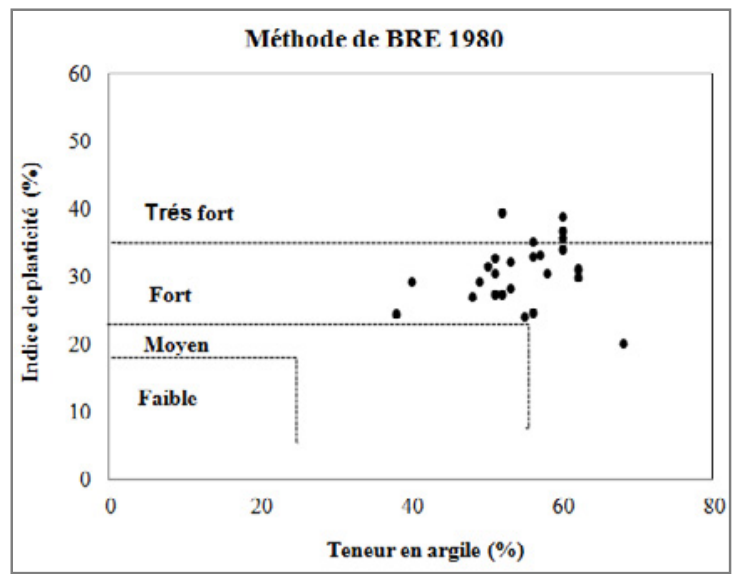

(d) : BRE, Royaume uni (1980)

Fig. 5. Méthode de classification à partir des essais d'identification.

Tableau 2. Potentiel de gonflement des sols examinés selon les différentes classifications

\begin{tabular}{|c|c|c|}
\hline $\begin{array}{c}\text { Méthode d'estimation du } \\
\text { potentiel de gonflement }\end{array}$ & $\begin{array}{c}\text { Paramètres de } \\
\text { classification }\end{array}$ & $\begin{array}{c}\text { Potentiel de } \\
\text { gonflement }\end{array}$ \\
\hline Seed et al.2, 1962 & AC, $\%<2 \mu \mathrm{m}$ & Élevé \\
\hline D'après Snethen (1984) & $\mathrm{I}_{\mathrm{p}}$ & Élevé \\
\hline D'après Chen (1988) & $\begin{array}{c}\text { WL }(\%), \% \\
<74 \mu \mathrm{m}\end{array}$ & Élevé \\
\hline
\end{tabular}

\begin{tabular}{|c|c|c|}
\hline $\begin{array}{c}\text { D'après l'etablissement } \\
\text { de recherche en bâtiment } \\
(1980) \text { (BRE. Royaume } \\
\text { uni) }\end{array}$ & Ip , $\%<2 \mu \mathrm{m}$ & $\begin{array}{c}\text { Élevé à très } \\
\text { élevé }\end{array}$ \\
\hline $\begin{array}{c}\text { Komornik et David et } \\
\text { Vijayvergiya et Ghazzaly } \\
(1973)\end{array}$ & WL, Ip & Élevé \\
\hline $\begin{array}{c}\text { Williams et Donaldson } \\
(1980)\end{array}$ & Ip $\%<2 \mu \mathrm{m}$ & Moyen à élevé \\
\hline Yukselen et Kaya (2008) & VB & Moyen \\
\hline
\end{tabular}

\subsection{Méthode directe}

Dans la mesure directe, trois paramètres sont utilisés pour qualifier le gonflement : le taux de gonflement ou gonflement libre, défini par $\mathrm{A}_{\mathrm{g}}=\mathrm{dh} / \mathrm{ho}$, la pression de gonflement $\mathrm{P}_{\mathrm{g}}$, pression qu'il faut appliquer à l'échantillon de sol pour empêcher son gonflement lors de l'hydratation et l'indice de gonflement $\mathrm{C}_{\mathrm{g}}$ qui traduit l'importance de la déformation de gonflement induit par un déchargement par rapport à un état de contraintes donné.

A partir de carottes d'argile extraites du site de Médéa, des échantillons cylindriques sont taillés et leurs faces sont dressées de façon à obtenir des surfaces planes, parallèles entre elles et perpendiculaires à l'axe de l'échantillon. Le diamètre est de $63,5 \mathrm{~mm}$ et la hauteur varie entre 25,4 et 20,4 mm. Deux modes d'essai de gonflement sont utilisés :

Pour la méthode de gonflement libre [1], Une fois l'échantillon est taillé, il est placé dans la cellule oedométrique sous une pression verticale correspondante au poids de la pierre poreuse et du piston, l'éprouvette est ensuite soumise à la contrainte des terres au repos jusqu'à stabilisation des déformations. Cette charge est enlevée et l'éprouvette est mise au contact de l'eau (Fig. $6)$.

Dans la méthode à volume constant [1], la déformation verticale est empêchée par l'application d'un chargement croissant d'une manière à garder constante la hauteur initiale de l'échantillon jusqu'à ce que l'échantillon ne présente plus de tendance au gonflement.

La norme ASTM est caractérisée par la correction de l'effet de remaniement de l'échantillon en lui appliquant une contrainte égale à la charge des terres au repos $\sigma_{\mathrm{V} 0}$ (fig. 6). L'évolution du gonflement unidimensionnel est appréciée à l'aide d'un capteur de déplacement au 1/1000 mm. Les mesures sont enregistrées électroniquement, à des intervalles de temps croissants. 


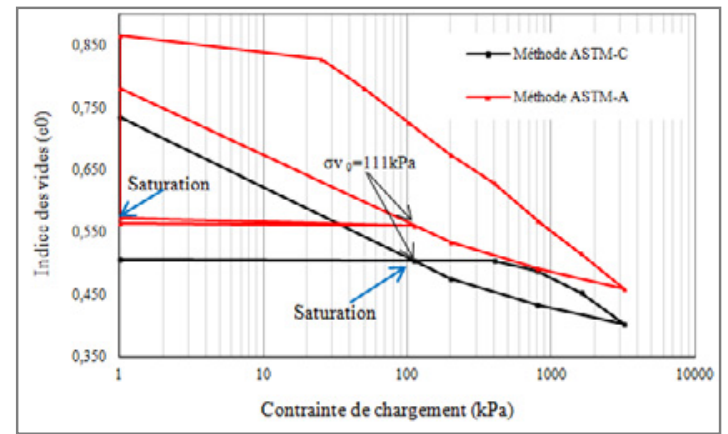

Fig. 6 Exemple des courbes de la norme ASTM (méthode A et C) réalisé sur E5 5,0/5,50 m

La figure 7 récapitule les différents résultats d'essais de gonflement à l'oedomètre. On note sur cette figure que la pression, le taux et l'indice de gonflement varient respectivement entre 100 et $900 \mathrm{kPa}, 5$ et $22 \%$ et 4 et 11 $\%$. La pression de gonflement est élevée d'après [6]. Selon [7], le taux de gonflement est élevé $(\mathrm{Ag}>5 \%)$ dans ce site, les valeurs de $\mathrm{Cg}$ indiquent un potentiel de gonflement moyen à élevé. l'ensemble de ces résultats sont en concordance avec ceux obtenus à partir des méthodes de classification, tenant en compte la surestimation du potentiel de gonflement par quelques méthodes vu quelles ne prennent pas en considération l'état initial représenté par la teneur en eau ainsi que la densité des échantillons.

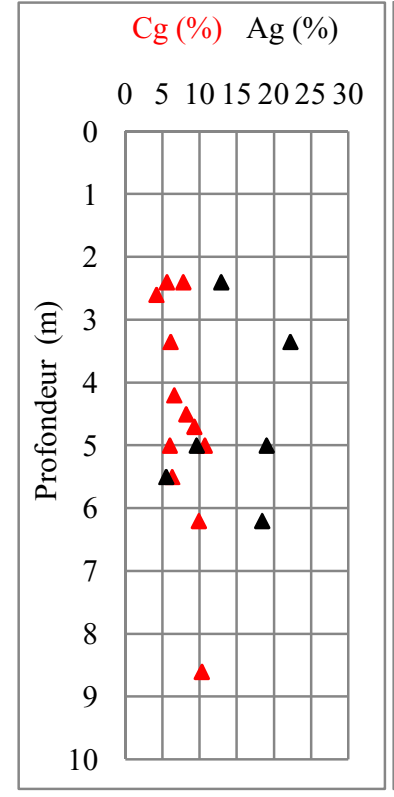

(a)

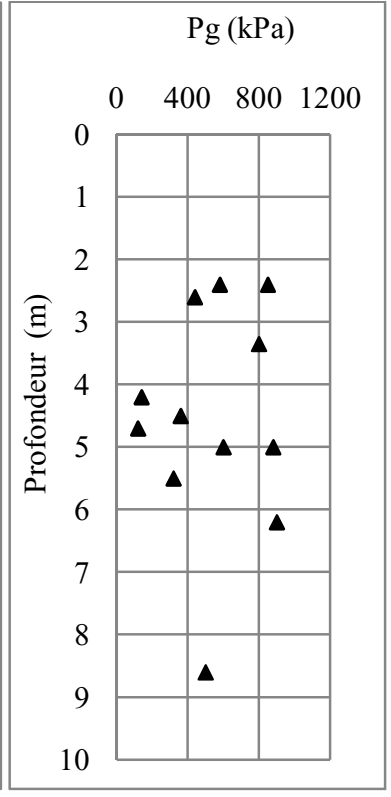

(b) (a) : Amplitude et indice de gonflement ; (b) : pression de gonflement

Fig. 7. Les résultats d'essais de gonflement sur les argiles de Médéa

\section{Mécanisme de gonflement}

Le gonflement se produit progressivement en fonction de l'évolution de la saturation dans la structure argileuse le long de la profondeur de la couche de cette formation. Le gonflement interfoliere se produit après le gonflement interparticulaire, l'eau pénètre dans la structure argileuse grâce à la création d'un réseau d'interstices dans le sol argileux au cours de l'inondation, ce qui fait augmenter l'indice des vides.

La déformation volumique dépend relativement de la quantité d'argile gonflante existante dans le sol, les argiles de type smectites absorbent une quantité importante d'eau vu la force d'attraction appliqué sur la particule de l'eau par la surface négative de ces argiles. Afin d'avoir plus d'information sur le comportement des argiles étudiées après saturation, des essais de gonflement libre ont été effectués selon la norme ASTM (fig. 8)

La courbe obtenue montre que le gonflement se produit suivant trois phases :

Phase d'initialisation : Sous l'effet des forces chimiques, la partie sèche de l'échantillon attire les particules d'eau ce qui engendre par la suite un écoulement dans les interstices. Cette phase sert à préparer les pores de la partie superficielle du sol à entamer le gonflement interparticulaire. Une fois ces forces sont amorties, le gonflement interparticulaire commence à se produire et l'échantillon entame la phase de gonflement primaire

Phase de gonflement primaire : elle est caractérisée par le gonflement des surfaces des particules argileuses entourant les pores saturés ainsi que le gonflement des feuillets argileux qui se trouvent dans les particules argileuses. Dans cette phase, le gonflement interfoliaire se produit massivement et progressivement. Les feuillets d'argile attirent les particules d'eau, d'où la déformation volumique importante de l'échantillon. Le temps de gonflement primaire dépend de la granulométrie, de la nature des minéraux argileux et de la hauteur de l'échantillon.

Phase de gonflement secondaire : dans cette phase un amortissement de gonflement est enregistré, l'échantillon est complètement saturé et les petites déformations sont liées aux réarrangements des particules et la stabilisation des liaisons chimiques. Cette phase peut durer plusieurs jours.

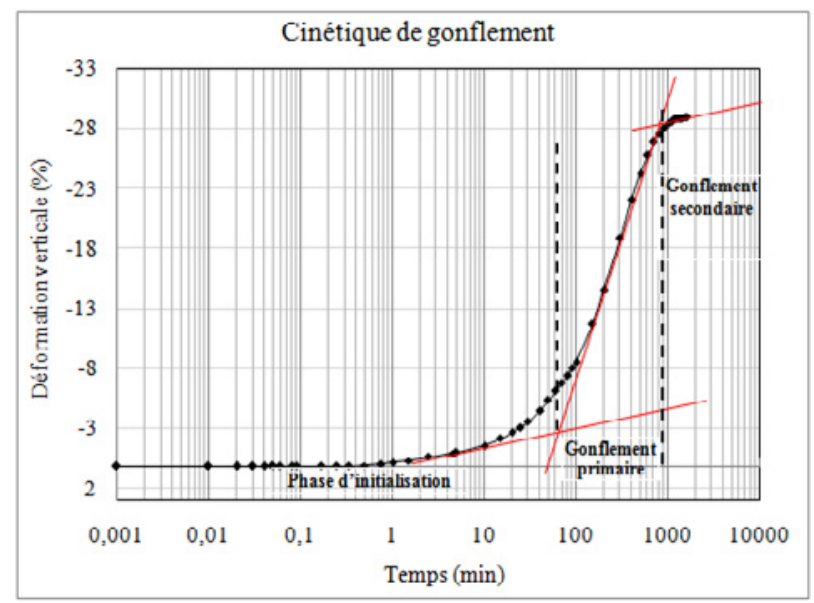

Fig. 8. Exemple de courbe de gonflement réalisée sur l'argile de Médéa

Des essais d'observation de l'évolution de la structure de l'échantillon au cours de gonflement après plusieurs cycles de séchage humidification ont été réalisés. $\mathrm{La}$ 
procédure d'essai consiste à exposer dans une enceinte climatique des échantillons initialement intacts à l'air sec sous une température de $35^{\circ} \mathrm{C}$ jusqu'à une teneur en eau constante puis à des conditions très humide qui permettent l'humidification de cet échantillon sous une température de $0^{\circ} \mathrm{C}$. La figure 9 montre l'évolution de la structure macroscopique après cinq cycles de séchagehumidification.

Après saturation complète de l'échantillon, on observe une augmentation considérable $\mathrm{du}$ volume de l'échantillon particulièrement dans le sens verticale. Lors du séchage, un rétrécissement important est observé dans le sens horizontal, ainsi qu'une apparition de fissures horizontales d'ordre de $1 \mathrm{~mm}$, ces fissures se referment suit à la saturation et réapparaissent après séchage.

A partir du premier cycle de séchage humidification l'échantillon d'argile perd sa densité initiale. Un réseau poreux est crée ce qui accélère la saturation de cet échantillon dans les cycles qui suivent. Un réarrangement de la structure superficiel s'est produit dans les trois premiers cycles et il s'est stabilisé à partir du troisième cycle (fig. 7 (f) et $(\mathrm{g})$ ) cette procédure à été appliquée sur trois d'autres échantillons de mêmes caractéristiques que celui montré dans la figure 9.

De cette étude on constate que la structure argileuse superficielle cherche son équilibre au cours de l'inondation et une fois atteint la structure se stabilise.

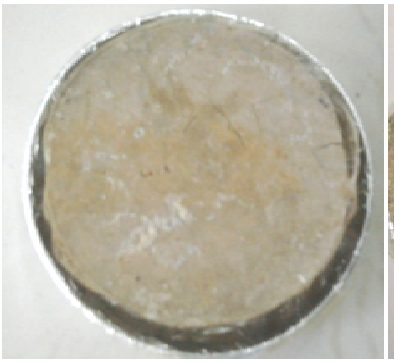

(a) initial

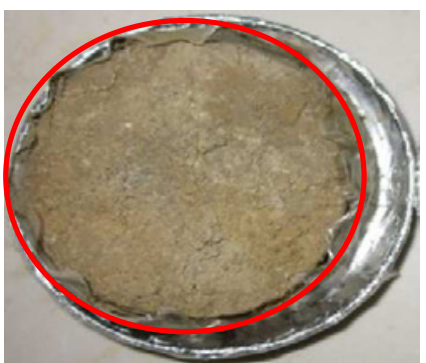

(c) Séchage (1 cycle)

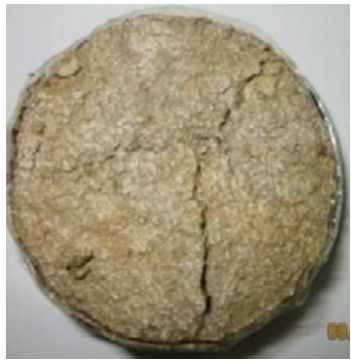

(e) Humide (3 cycles)

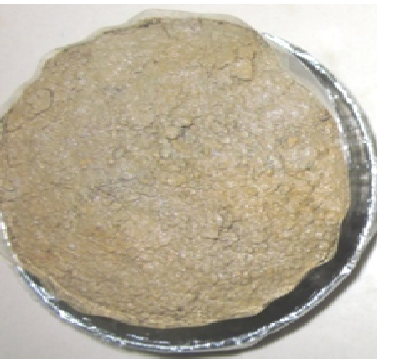

(b) humide (1 cycle)

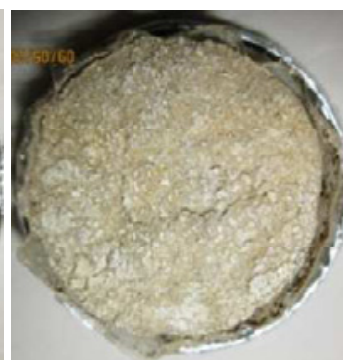

(d) Sèche (2 cycles)

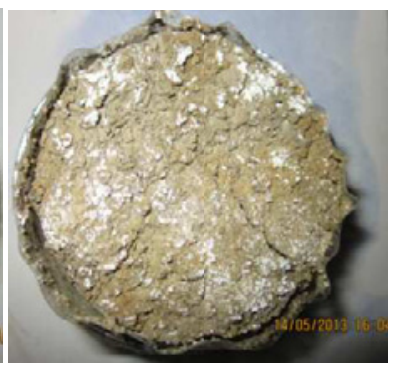

(f) Séchage (3 cycles)

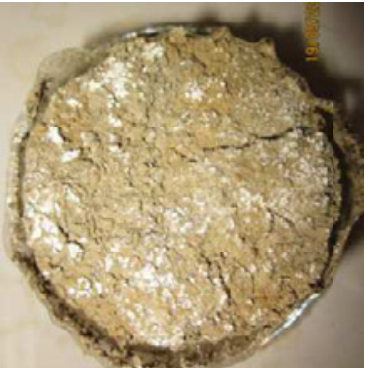

(g) Séchage (4 cycles)

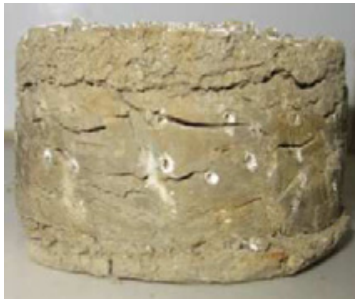

(i) vue verticale (5 cycles)

(j) la différence avec l'état l'initial

Fig. 9. Évolution de la structure argileuse après des cycles de séchage humidification, échantillon du site de Médéa

\section{Conclusion}

La région de Médéa est caractérisée par un climat chaud et $\mathrm{sec}$ en été et pluvieux et humide en hiver. Le site étudié consiste en une formation argileuse marneuse compacte. Les résultats des essais d'identification physiques et chimiques montrent que cette formation est très plastique et active $(\mathrm{Ac}>1,5)$. Les valeurs de la surface spécifique $\left(>100 \mathrm{~m}^{2} / \mathrm{g}\right)$ rentrent dans l'intervalle des sméctites qui sont des minéraux très gonflants.

La majorité des méthodes de classification basées sur les essais physique et chimique estiment que le site étudié est d'un potentiel de gonflement élevé. Ce résultat est en concordance avec les résultats des essais de gonflement. Les observations après plusieurs cycles de séchagehumidification montrent que le sol argileux de Médéa est en mouvement continuel conditionné par la variation de la teneur en eau, ce sol gonfle en hiver à cause de l'importance de la pluviométrie et d'humidité et rétrécit en été vue l'importance des valeurs de la température et de l'évaporation. On constate aussi que ce mouvement se déroule avec un rythme constant après un certain nombre de cycles de séchage - humidification.

\section{Références bibliographiques}

1. ASTM Standard test methods for one-dimensional swell or settlement potential of cohesive soils, ASTM D 4546-85, (1986)

2. A. Komornik, D. David, Prediction of swelling pressure of clays. Proc ASCE, Journal of Soil Mechanics and Foundation Engineering Divison, ASCE, Vol. 95, n²1, pp 209- 225 (1969)

3. A.B. Williams \& G.W. Donaldson, Developments related to building on expansive soils in South Africa. In: Proc 4th Int Conf On Expansive soils, Denver 2:834-844 (1980) 
4. A. Djedid, identification et prévision du gonflement de quelques solsde la région de Tlemcen (Algérie), bulletin des laboratoires des ponts et chaussées $n^{\circ}$ 233 réf. 4375, pp. 67-75 (2001)

5. Building Research Establishment, Low-rise buildings on shrinkable clay soils: Part 1. BRE Digest 240, HMSO, London, (1980)

6. F. H. CHEN, S. MA G. Swelling and shrinking behaviours of expansive clays, International Conference on expansive soils, New Delhi, pp 127129, (1987)

7. H.B. Seed, R.J. Woodward \& R.de Lundgren, Prediction of swelling potentiel for compacted clays. J. Soil Mech and Found Div. ASCE, 88:107-131, (1962)

8. T. N Lan, Nouvel essai d'identification des sols : l'essai au bleu de méthylène. Bull LPC 88:136-137 (1977)

9. Y. Yukselen \& A. Kaya, Suitability of the méthyléne blue test for surface area: cation exchange capacity and swell potential determination of clayey soils. Engineering Geology 102:38-45, (2008) 